\title{
Effects of elevated vacuum on in-socket residual limb fluid volume: Case study results using bioimpedance analysis
}

\author{
Joan E. Sanders, PhD; ${ }^{*}$ Daniel S. Harrison, BS; Timothy R. Myers, MME; Katheryn J. Allyn, LCPO \\ Department of Bioengineering, University of Washington, Seattle, WA
}

\begin{abstract}
Bioimpedance analysis was used to measure the residual limb fluid volume of seven transtibial amputee subjects using elevated vacuum sockets and nonelevated vacuum sockets. Fluid volume changes were assessed during sessions with the subjects sitting, standing, and walking. In general, fluid volume losses during 3 or 5 min walks and losses over the course of the $30 \mathrm{~min}$ test session were less for elevated vacuum than for suction. Numerous variables, including the time of day that data were collected, soft tissue consistency, socket-tolimb size and shape differences, and subject health, may have affected the results and had an equivalent or greater effect on limb fluid volume compared with elevated vacuum. Researchers should well consider these variables in the study design of future investigations on the effects of elevated vacuum on residual limb volume.
\end{abstract}

Key words: accommodation, amputee, diurnal, elevated vacuum, lower limb, prosthetic fit, prosthetics, residual limb, suction, volume change.

\section{INTRODUCTION}

Changes in residual limb volume over time are an important challenge for people who use prosthetic limbs. Residual limb volume changes, both diurnal and longterm, can cause the fit of the prosthetic socket to change. If the residual limb reduces in volume, then the socket becomes loose and bony prominences are subjected to greater stress, potentially causing pain and increasing risk of injury. If the residual limb increases in volume, then socket pressures on the limb will increase and blood flow can be restricted, limiting nutrient delivery and causing a buildup of cell waste in the tissues. Thus, a technology that controlled limb volume and kept it stable might overcome these problems and reduce pain and injury in people using prosthetic limbs.

Elevated vacuum, also termed vacuum assist, was introduced in the prosthetics industry approximately 15 years ago with an aim of reducing volume loss in the residual limb over time [1]. An elevated vacuum system attaches to a prosthesis and draws a vacuum at the distal end of the socket. Particularly during swing phase, the vacuum pulls residual limb soft tissues outward, thereby lowering pressure within the interstitial fluid inside the residual limb. The reduction in interstitial fluid pressure increases the arterial to interstitial pressure gradient while decreasing the venous to interstitial pressure gradient. The result may then be an increase in the amount of fluid leaving the arterial vasculature into the interstitial space and a decrease in the amount of fluid moving from the interstitial space into the venous system [2]. The net result is then a reduction in fluid loss out of the residual limb, i.e., less residual limb volume reduction. Thus, elevated vacuum may serve to reduce limb fluid volume

\footnotetext{
Abbreviation: $\mathrm{PTB}=$ patellar tendon bearing.

*Address all correspondence to Joan E. Sanders, PhD; University of Washington-Bioengineering, 355061, Foege N430J, 3720 15th Ave NE, University of Washington, Seattle, WA 98195; 206-221-5872; fax: 206-685-3300.

Email: jsanders@u.washington.edu

DOI:10.1682/JRRD.2010.11.0219
} 
loss. This effect would be beneficial to people who would otherwise lose limb fluid volume over the day.

Studies have been conducted to investigate transtibial amputee limb volume changes using elevated vacuum systems [3-5]. Board et al. reported limb volume differences over the course of a 30 min walking session by measuring predonning and postdoffing limb volumes [3]. They compared results using sockets with elevated vacuum versus sockets without vacuum (suction). All 10 subjects tested had their amputation as a result of traumatic injury or were congenital amputees. The tests for each subject were conducted on the same day, and the same socket was used for both tests. Results showed that all residual limbs underwent volume reduction for the novacuum (suction) condition. The mean volume reduction was 6.5 percent. Nine of ten subjects underwent volume enlargement for the with-vacuum condition. The mean volume increase was 3.7 percent. Goswami et al. extended from these studies to investigate residual limb volume changes after $30 \mathrm{~min}$ of walking with three differently sized sockets: 4 percent (by volume) undersized, optimally sized, and 4 percent oversized [4]. Though who defined "optimally sized" and how are unclear, the undersized and oversized designs were achieved with computer-aided manufacturing methods and were estimated to be accurate to within \pm 2 percent volume. Eleven subjects were enrolled, though only seven subjects were tested with all three sockets. Use of undersized sockets resulted in a mean volume loss of 1.8 percent from the day's volume baseline. Use of optimally sized sockets resulted in a mean volume gain of 7.0 percent from the day's baseline. Use of oversized sockets resulted in a mean volume gain of 12.9 percent from the day's baseline. These results suggest that elevated vacuum can increase limb volume if the socket is sufficiently large. In a single-subject study, Gerschutz et al. showed that percent absolute volume changes in the residual limb over a 10 min period after walking and doffing the socket were less after an elevated vacuum socket was worn than after a suction socket was worn [5].

Though Board et al., Goswami et al., and Gerschutz et al. reported the first data in the literature testing elevated vacuum sockets [3-5], the researchers did not measure in-socket limb volumes. Board et al. and Goswami et al. casted each subject's residual limb in alginate before donning and after doffing, then filled the casts with water to determine limb volume [3-4]. They determined volume differences over a session by comparing the predonning with the postdoffing alginate cast volumes [3] or the day's baseline volume with that measured postdoffing [4]. Gerschutz et al. measured pre- and postdoffing volumes by using a laser scanner on the subject's residual limb while the subject wore an elastomeric liner [5].

The purpose of our study was to extend from the previous out-of-socket investigations to determine whether an in-socket volume measurement technique produced results consistent with previous findings. A series of case studies are presented. Using bioimpedance analysis, we measured extracellular fluid volume changes on individuals with transtibial amputation while they ambulated with either elevated vacuum sockets, suction sockets, or sockets with lock-and-pin suspension. For bioimpedance results in the present investigation to be consistent with previous findings, we would expect that limb fluid volume would be maintained or would increase during walking when elevated vacuum was used. We would also expect that for a high vacuum pressure compared with a low vacuum pressure or compared with a lock-and-pin suspension socket, limb fluid volume would decrease less (or increase more) during walking, cyclic (within-step) fluid volume changes during walking would reduce (because of improved suspension), and limb fluid volume would decrease less (or increase more) over $30 \mathrm{~min}$ test sessions.

\section{METHODS}

\section{Subjects}

Seven subjects with unilateral transtibial amputation participated in this study. All had their amputation for at least $1 \mathrm{yr}$ and could walk comfortably without assistive devices for at least a 5 min period. All were fitted by certified prosthetists trained in elevated vacuum socket design. During data collection sessions, all socket fits were deemed by the research prosthetist (coauthor) to be acceptable for regular use.

\section{Bioimpedance Analysis}

Bioimpedance analysis is a technique commonly used to assess body composition/body fat [6-11] as well as fluid imbalance in hemodialysis patients [12-15]. Bioimpedance takes advantage of the difference in response of different biological structures to electrical current. Current will readily pass through cell-free biological 
fluid at all frequencies between approximately $5 \mathrm{kHz}$ and $1 \mathrm{MHz}$. However, it will easily penetrate cell membranes only at high frequencies, not at low frequencies. Thus, by applying current across a range of frequencies to a limb segment, measuring voltage potential in a section of the limb within the electric field, and then using a computational model to process the data [16], one can determine extracellular and intracellular fluid volumes [17].

\section{Volume Measurement}

Residual limb extracellular fluid volume was measured with use of bioimpedance analysis. A low current was applied between two outer pair electrodes on the residual limb while voltage was measured between two inner pair electrodes (Figure 1). The electrical current $(<700 \mu \mathrm{A})$ was applied at 50 frequencies between $5 \mathrm{kHz}$ and $1 \mathrm{MHz}$ with a commercial device (Hydra 4200, XiTRON Technologies; San Diego, California). One set of frequencies was sampled each second. Because cell membranes have a high capacitance, at low frequencies, current passes primarily through extracellular tissues, while at high frequencies, it passes through both extracellular and intracellular tissues. Bone and adipose tissue are minimally conductive, while skin and muscle are highly conductive. Thus, bioimpedance data reflect primarily fluid changes within skin and muscle [18]. The collected data, amplitude and phase change at 50 frequencies, were then fitted to an electrical analog model [17] (Cole model) to determine extracellular fluid resistance. With use of the Cole model, tissue was modeled as an extracellular resistance in parallel with a cell membrane capacitance and intracellular resistance. Nonlinear weighted least squares curve-fitting applied to the multifrequency impedance spectrum was used to extrapolate extracellular fluid resistance and total fluid resistance at the lowand high-frequency limits. Resistance data were then converted to fluid volume data through a limb segment model [19].

Bioimpedance is a very sensitive measure of limb fluid volume, and no accepted gold standard with better resolution exists with which to compare it. However, comparison with lower-resolution volume measurement techniques has shown it to correlate well with deuterium oxide and bromide dilution techniques [7,20-23] as well as with magnetic resonance imaging [24].

In this article, only extracellular fluid volume data are presented. Extracellular fluid volume is likely the primary source of fluid volume changes within the residual

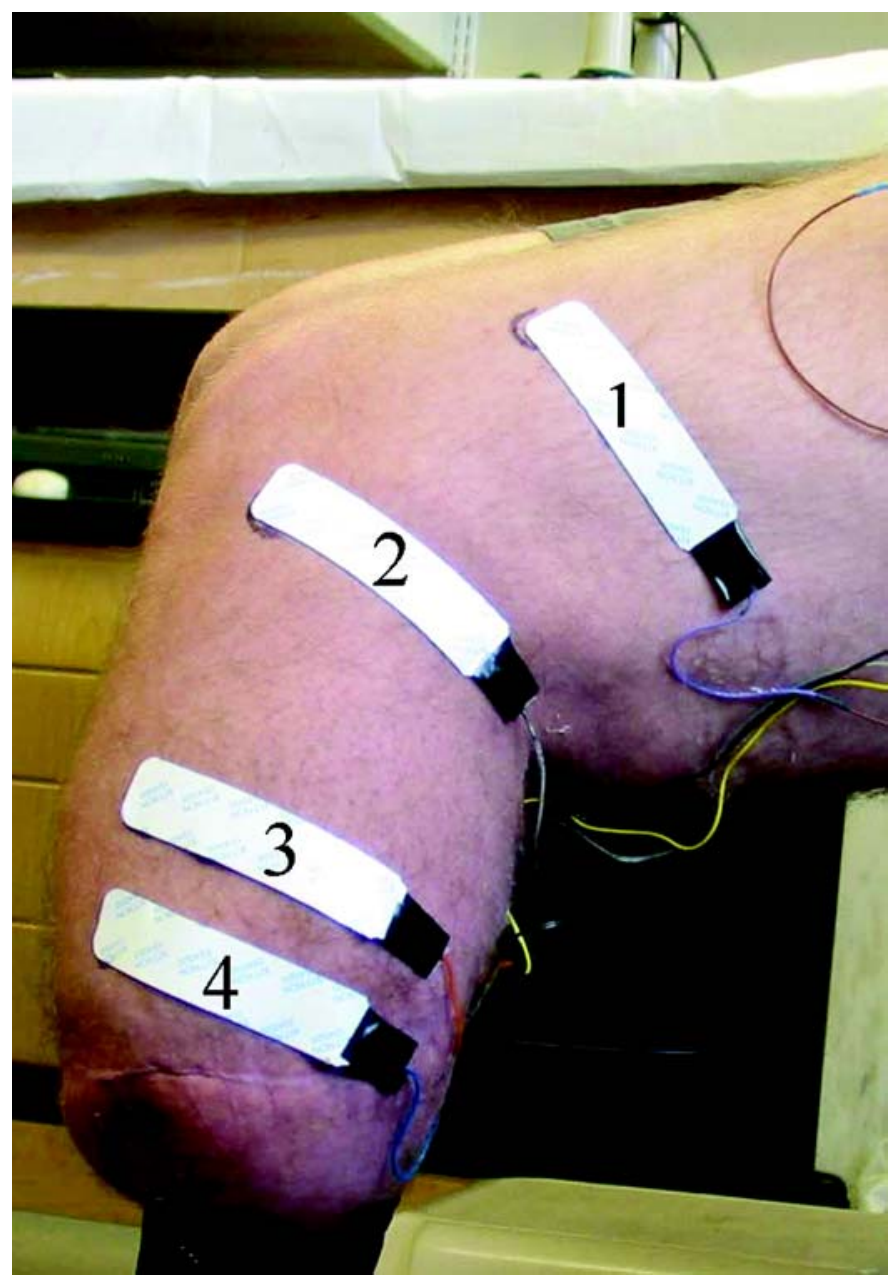

\section{Figure 1.}

Electrodes positioned on subject's residual limb. Outer electrodes 1 and 4 were current injecting while inner electrodes 2 and 3 were voltage sensing. Wires to electrodes were strain-relieved using Tegaderm (3M; St. Paul, Minnesota).

limb over short-term intervals ( $<40 \mathrm{~min})$. Intracellular fluid transport would be expected to be too slow to accomplish significant volume change within the approximately 30 to $40 \mathrm{~min}$ long test sessions conducted here.

Because the electrodes were within the prosthetic socket during testing, the standard alligator clips used to connect the XiTRON Technologies instrument to the electrodes could not be used. A custom four-pin Delrin connector designed to accommodate gold-plated pins (WPI Viking, Cooper Interconnect; Chelsea, Massachusetts) was developed to attach four 28-gauge insulated lead wires from the XiTRON Technologies instrument cable to 
the electrodes. Each wire was strain-relieved and mechanically stabilized at the electrode tab connection by looping of the wire onto the tab, light soldering of the exposed end to the tab, and then placement of a polystyrene disk (9 $\mathrm{mm}$ diameter, $0.25 \mathrm{~mm}$ thickness) over the solder connection. The tab was then covered with a single layer of vinyl electrical tape (Super 88, Scotch, 3M; St. Paul, Minnesota). A $9 \times 26 \mathrm{~mm}$ section was cut from the adhesive part of a Band Aid (Johnson \& Johnson; New Brunswick, New Jersey). The outer surface of the Band Aid was glued (Skin-Bond Cement, Smith \& Nephew; St. Petersburg, Florida) onto the outside of the electrical tape so that the Band-Aid's sticky surface was exposed. The Band-Aid's sticky surface was put on the skin so that, like the electrode, it stuck to the skin. This attachment method was necessary to avoid excessive strain application to the tabsolder connection that otherwise would have caused mechanical damage and failure of the electrode.

\section{Protocol}

After informed consent was obtained, subjects were asked not to consume alcohol or caffeine on the day of testing before coming into the laboratory. After arriving at the laboratory, the subject walked briefly on a treadmill (Clubtrack, Quinton Instruments Company; Bothell, Washington) to determine a normal self-selected walking speed. The subject then sat quietly in a chair while basic subject information was collected: age, date of amputation, cause of amputation, health status, regular activities, recent limb health history, and recent changes to prosthesis. Then, the subject removed his or her prosthesis, and the research practitioner inspected the residual limb to ensure there were no sores or injury. The skin locations where electrodes were to be placed were cleaned by gentle rubbing with sandpaper (Red Dot ${ }^{\mathrm{TM}}$ Trace Prep 2236, $3 \mathrm{M})$. Sandpaper was used rather than an alcohol-based cleaning agent because alcohol dries the skin and reduces conductance [18]. Two current-conducting and two voltage-sensing strip electrodes (XiTRON Technologies) (77 $\times 20 \mathrm{~mm}$ contact surface, $0.81 \mathrm{~mm}$ thickness) were placed on the lateral posterior surface of the subject's limb such that the long axes of the electrodes were parallel to each other and perpendicular to the long axis of the residual limb (Figure 1). A thin layer of coupling gel (Couplant D, Panametrics, General Electric Company; Fairfield, Connecticut) was applied to the electrode before it was placed on the skin. The proximal voltagesensing electrode was at the level of the patellar tendon, proximal of the fibular head. The distal current-injecting electrode was positioned as far distally as possible but still on the relatively cylindrical portion of the residuum. The distal voltage-sensing electrode was positioned between 3.0 and $4.5 \mathrm{~cm}$ proximal to the distal currentinjecting electrode. The proximal current-injecting electrode was positioned on the thigh outside the proximal socket brim but beneath the liner or suspension sleeve. Lead wires were strain relieved on the posterior residual limb surface with use of rectangular pieces $(6.0 \times 3.5 \mathrm{~cm})$ of Tegaderm (3M). So that vacuum within the socket was maintained, a piece of Tegaderm was also placed over the lead wires at the proximal edge of the suspension sleeve. As long as the Tegaderm was placed flat on the skin and over each lead wire individually, air did not enter along the edges of the lead wires into the socket. During each test session, the research practitioner carefully inspected the socket to ensure vacuum was well-maintained.

For subjects using a manual elevated vacuum system (Harmony, Otto Bock; Minneapolis, Minnesota), the unit was modified so that the vacuum could be easily connected or disconnected during the session. This capability was achieved by placement of a stopcock in series in the plastic tubing connecting the mechanical pump to the valve in the socket. By adjusting the stopcock orientation, the research practitioner could set either a vacuum or a suction condition. For the electronic elevated vacuum systems, no such modification was necessary because they were powered by an adjustable electronic pump. The vacuum level was increased or decreased with switches on the vacuum unit.

Data collection involved periods of sitting, standing, and walking. For the sitting portion of the protocol, the height on the subject's chair was adjusted so as to maintain the knee in $100^{\circ}$ to $140^{\circ}$ of extension of extension with the foot resting on the floor. During the standing portions of the protocol, the subject stood on a $6.4 \mathrm{~cm}$ high platform with an electronic weight scale (349KLX HealthO-Meter, Pelstar LLC; Alsip, Illinois) embedded within it so that the top of the scale was flush with the surface. Weight bearing on the limb instrumented with the bioimpedance electrodes was monitored at a $2 \mathrm{~Hz}$ sampling rate and observed by one of the researchers on a computer screen. If the weight on the scale deviated by more than 10 percent of half the subject's body weight, then the subject was instructed by the research practitioner to shift his or her weight to the appropriate leg to achieve more equal weight bearing. A 10 percent threshold was used because 
in preliminary investigations we determined that this range of weight-bearing change did not typically induce changes in bioimpedance results and it did not typically necessitate continual instruction to the subject for weight shifting. In preliminary investigations, continual weight shifting caused some subjects to stiffen up, distorting the data of interest. During the walking segments, the subject walked on the treadmill at the speed established at the outset of the session as described. Subjects walked for 3 or 5 min periods (depending on the protocol) on the treadmill. A 3 or 5 min period was selected because all subjects could achieve those durations without fatigue. Further, we desired to change socket vacuum conditions during tests but not prolong the entire test to more than 40 min total.

Custom MATLAB code (v. 7.4, The MathWorks; Natick, Massachusetts) was written so as to display the limb fluid volume data to the researchers in essentially real time (3 s delay). This display was essential during data collection to ensure subjects did not occlude a major blood vessel during sitting (apparent as a rapid change in limb fluid volume during sitting) and to ensure equipment was functioning properly throughout the session. Clinicians on the research team found the immediate data presentation helpful toward clinical interpretation. Data from the electronic scale sampled at $2 \mathrm{~Hz}$ were collected simultaneously with the bioimpedance data, using the same computer as used to display bioimpedance data. We put time stamps in the code so that bioimpedance data could be lined up with subject weight-bearing data during standing.

\section{Data Processing and Analysis}

After the session was completed, software provided by the manufacturer (version 2.2, XiTRON Technologies) was used to process the collected bioimpedance data. The software used the Cole model approach as described in the literature [17], optimizing a nonlinear least squares error of magnitude and phase to determine extracellular fluid resistance. To avoid deleting data points solely to force fit the Cole model, data at a frequency was removed from analysis only if including it decreased the total weighted least squares error with specified limitations. The data were then converted to extracellular fluid volume with use of a well-accepted model [19] and then expressed as a percentage of the extracellular fluid volume measured at the end of the initial 2 min sit interval (prosthesis donned).

Later during postprocessing, to facilitate inspection of the data, a 10-point moving mean of the percent change in extracellular fluid volume was plotted, shifted back 5 points to realign it with the original data. Because the sampling rate $(\sim 1 \mathrm{~Hz})$ was less than the walking frequency, measuring fluid volume change within each step was not possible. However, in this analysis, it was the change over the course of the 3 or 5 min walking interval that was of interest; thus, use of the moving mean curve in analysis was considered appropriate.

\section{RESULTS}

On the basis of previous studies testing the capabilities of bioimpedance analysis for lower-limb assessment [25], we considered 0.2 percent to be the lowest percentage fluid volume change that could be accurately resolved in this research. This resolution limit was principally due to bit quantification error in the instrument and processing algorithm. Thus, in the presentation below, differences in limb fluid volume change between two test conditions on a subject were considered meaningful only if they were greater than 0.2 percent.

\section{Case 1: Long-Term User of Electronic Elevated Vacuum Unit-Test Session Using Elevated Vacuum}

\section{Case 1}

This 42 yr old male had his left lower limb amputated 5 yr prior as a result of traumatic injury. His limb was revised 16 mo after his initial amputation because of complications from surgical staples within his residuum. His residual limb length was $16 \mathrm{~cm}$ from the midpatellar tendon to distal end and cylindrical in shape with a fair amount of hair. He was $180 \mathrm{~cm}$ in height, $88 \mathrm{~kg}$ in mass, and a K-4 level [26] ambulator. His prosthesis was a total contact socket design with an elevated vacuum unit and a dynamic response foot (Renegade Freedom Foot, Freedom Innovations; Irvine, California). He used elevated vacuum because of prior diurnal residual limb volume change problems using a Pelite liner and because he had an interest in trying elevated vacuum suspension. One year before our bioimpedance testing, he switched from a manual elevated vacuum system (Harmony, Otto Bock) to an electronic system (eVAC, Smith Global; Laurie, Missouri). In bioimpedance testing, he was tested with his electronic system. He had a history of blister problems when he set the vacuum pressure at the highest setting, 15 psi (104 kPa), instead of his usual setting, 10 psi (69 kPa). 
These pressure settings were the values labeled on the elevated vacuum unit; we did not measure the actual pressures. Thus, we do not know whether the stated pressure levels were achieved in the present study. The subject typically wore his prosthesis for $16 \mathrm{~h} / \mathrm{d}$ and wore a 5-ply and 3-ply sock outside of the liner. He did not add or remove socks during the day.

\section{Protocol}

Throughout the session, the vacuum was operated at the subject's normal vacuum setting. After sitting comfortably for $2 \mathrm{~min}$, the subject stood with equal weight bearing for $5 \mathrm{~min}$ and then walked on the treadmill at his preferred walking speed for 5 min. After sitting quietly for $2 \mathrm{~min}$, he again stood for $5 \mathrm{~min}$ with equal weight bearing and then walked on the treadmill for $5 \mathrm{~min}$. He then sat, doffed his prosthesis and liner, and sat quietly for $10 \mathrm{~min}$. Thus, the total session duration was approximately $34 \mathrm{~min}$.

We attempted to conduct additional sessions with reduced vacuum pressure settings during ambulation so as to evaluate the influence of vacuum pressure intensity on the bioimpedance measured. However, the subject was unable to walk comfortably under those conditions and had trouble maintaining adequate suspension.

\section{Results}

Results from this subject showed that limb fluid volume decreased during stands and increased during walks (Figure 2). The decreases during stands averaged 0.9 percent and the increases during walks averaged 1.5 percent. Thus, the increase from walking more than offset the decrease from the immediately prior standing $(1.5 \%>0.9 \%)$. The cyclic changes during the walks visible in the plot (Figure 2) were due to pistoning and/or deformation of the residual limb within the socket, and they are typical of reports in prior investigations [25,27]. Fluid volume gradually increased over the course of the session (beginning of 1st stand to end of 2nd walk) of 2.1 percent. The peak-to-peak change in fluid volume during walking averaged 2.0 percent. The fluid volume change during the 10 min period during sitting after doffing was 1.7 percent.

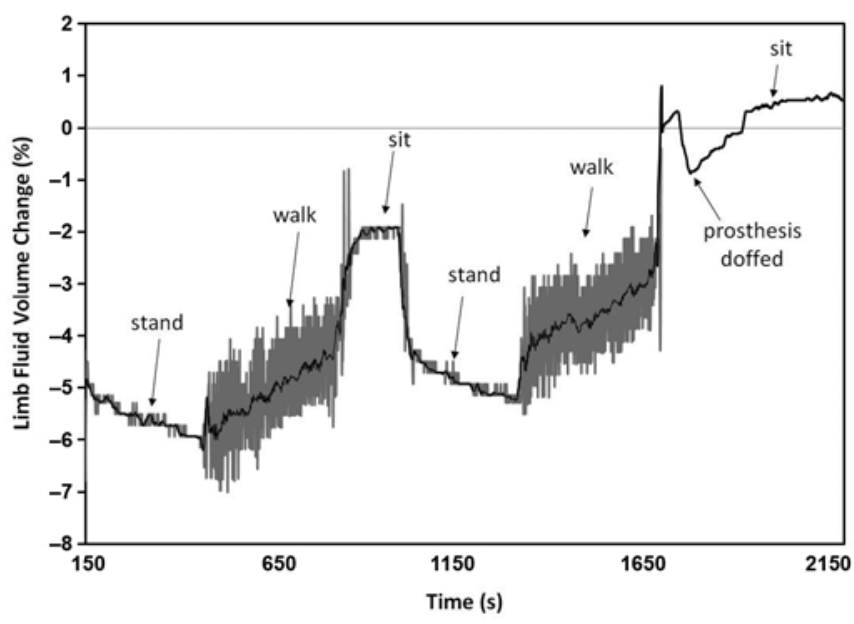

Figure 2.

Results from case 1. Gray line is original data, and black line is 10point moving mean. $0 \%$ represents baseline at outset of session 2 min after sitting quietly with prosthesis donned. This long-term elevated vacuum user showed increased limb fluid volume over session.

\section{Cases 2, 3, and 4: Short-Term Users of Manual Elevated Vacuum - Within-Session Comparison of Elevated Vacuum Versus Suction}

These subjects were short-term users (3-4 wk) of the manual (Harmony) system prior to bioimpedance testing. For each of the three subjects, a new socket was made to use with the elevated vacuum prosthesis. No locking pin was used with the elevated vacuum socket. All had residual limbs with prominent bony structures and little redundant soft tissue.

\section{Case 2}

This male subject was $61 \mathrm{yr}$ of age and had his amputation $5 \mathrm{yr}$ prior as a result of traumatic injury. His $17 \mathrm{~cm}$ long residual limb was conical in shape with a fair amount of hair and little soft tissue. He was $175 \mathrm{~cm}$ tall, $73 \mathrm{~kg}$ in mass, and a K-4 level ambulator. He was healthy and did not take any medications, though he used to be a smoker (for $20 \mathrm{yr}$; stopped smoking more than 5 yr ago) and his blood pressure was on the high end of normal. His regular prosthesis was a patellar tendon bearing (PTB) endoskeletal prosthesis with a gel liner and lockand-pin suspension, and his prosthesis was equipped with a dynamic response foot (Luxon Max, Otto Bock). He wore his prosthesis for at least $16 \mathrm{~h} / \mathrm{d}$. He typically did not add socks to compensate for limb volume change except on days when he was very active. He was a good 
candidate for elevated vacuum because of his high level of activity, desire for optimal proprioception, and need for fewer sock changes to eliminate pistoning during active days. Before bioimpedance testing, he was fitted with a new socket equipped with a P2 Harmony vacuum unit and a Seattle Lightfoot (TruLife; Dublin, Ireland). He used this prosthesis exclusively for the $3 \mathrm{wk}$ before bioimpedance testing.

\section{Case 3}

This subject was a 48 yr old male and had his amputation 24 yr prior as a result of traumatic injury. His $19 \mathrm{~cm}$ long residual limb was conical in shape with a fair amount of hair and very little soft tissue. He was $188 \mathrm{~cm}$ in height and $80 \mathrm{~kg}$ in mass. A K-4 level ambulator, this subject was healthy, did not take medications, and used his prosthesis for at least $12 \mathrm{~h} / \mathrm{d}$. He regularly used a PTB endoskeletal prosthesis, a flexible socket liner with a gel liner, a lock-and-pin suspension, and a Freedom Innovations dynamic response prosthetic foot. He had a history of ingrown hairs/blisters that formed when his prosthesis pistoned excessively as a result of limb volume changes. His limb typically changed volume after high activity. He was a good candidate for elevated vacuum because of his high activity, mechanical aptitude, need for superior suspension, and desire to not add socks throughout the day to compensate for volume fluctuations. Before bioimpedance testing, he was fitted with a new socket equipped with a P2 Harmony vacuum unit and a Seattle Lightfoot. He used this prosthesis exclusively for the 3 wk before bioimpedance testing.

\section{Case 4}

This subject was a 54 yr old male who had a transtibial amputation 4 yr prior as a result of traumatic injury. His residual limb was $23 \mathrm{~cm}$ in length, conically shaped, with good hair and sensation. He had a distal neuroma removed 4 mo before bioimpedance testing. He was $188 \mathrm{~cm}$ in height, $77 \mathrm{~kg}$ in mass, and a K-3 level ambulator. He used his prosthesis for approximately $16 \mathrm{~h} / \mathrm{d}$. He had a history of smoking and high cholesterol and had been diagnosed with peripheral arterial disease and peripheral vascular disease. He had pain in his calf when ambulating more than two blocks. Because of volume fluctuation problems in his limb, he typically added socks during the day. In an effort to enhance suspension and reduce volume fluctuation problems, 4 wk before bioimpedance testing, his regular prosthetist switched him from a PTB endoskeletal prosthe- sis with a gel sock, Pelite liner, and neoprene suspension to a manual elevated vacuum system (P2 Harmony). He used this manual elevated vacuum socket during bioimpedance testing, with a dynamic response Flex-Foot (Össur Americas; Foothill Ranch, California).

\section{Protocol}

For these subjects, part of the trial was conducted with the vacuum off (suction) and part with it on (elevated vacuum). The protocol was started with the stopcock set for a suction socket. After 2 min of sitting, the subject stood with equal weight bearing for $3 \mathrm{~min}$. He then walked on the treadmill for $3 \mathrm{~min}$ at his nominal walking speed. The subject then stopped, and the stopcock was turned so as to apply elevated vacuum to the prosthesis. The subject then walked on the treadmill again for $3 \mathrm{~min}$. After 2 min of sitting, the subject stood and walked for 3 min each, still with the elevated vacuum. The stopcock was then switched back to a suction socket, and the subject again walked for 3 min. Walking intervals of 3 min duration were used in these studies rather than 5 min because of concern that subjects would experience discomfort using the heavy Harmony system without elevated vacuum. We were concerned that subjects would not be able to complete 5 min treadmill walking with suction alone. Also, using this ordering (walk without elevated vacuum, walk with elevated vacuum, sit, walk with elevated vacuum, and walk without elevated vacuum), we would be able to distinguish a continuous increase or decrease in limb fluid volume over the session from influence of elevated vacuum. For cases 2 and 3 , instrumentation problems occurred during the last walk; thus, data from only the first two walks were included in the analysis described below.

\section{Results}

None of the three subjects demonstrated the continuous rise in limb fluid volume during walking that case 1 did. Fluid volume tended to increase and then plateau during walks when the vacuum was activated for cases 2 and 3 (Figure 3). For case 2, limb fluid volume increased 1.2 percent during walking with the vacuum activated while for case 3, it increased 0.4 percent (Table 1, column 3). For case 4, limb fluid volume decreased 0.5 percent during the first walk with vacuum activated and 0.7 percent during the second walk with the vacuum activated, for an average decrease of 0.6 percent. 
JRRD, Volume 48, Number 10, 2011

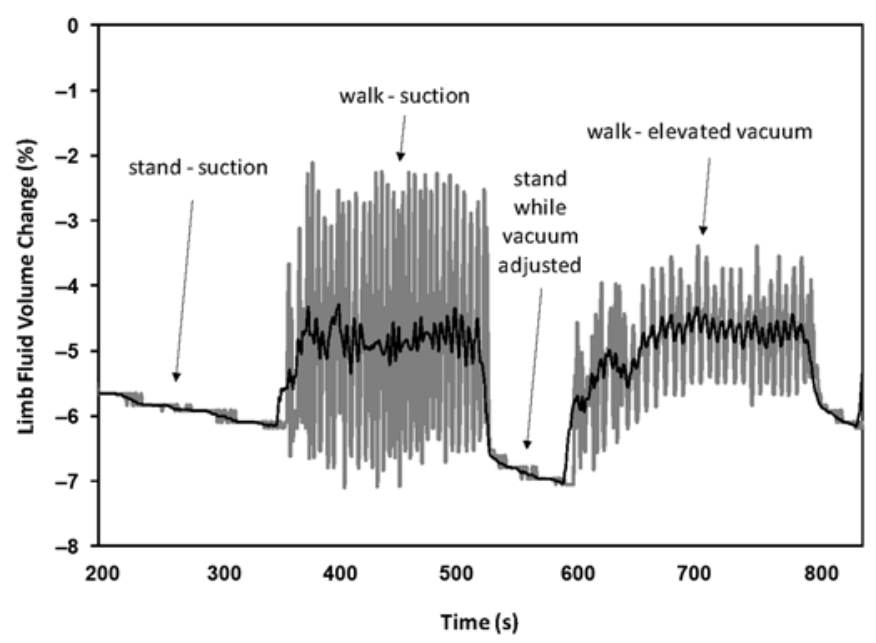

Figure 3.

Switching from suction to elevated vacuum (Harmony, Otto Bock; Minneapolis, Minnesota). Results from case 2 for part of trial are shown. Vacuum was switched from suction to elevated vacuum at approximately $550 \mathrm{~s}$.

Limb fluid volume after walking 3 min with suction was comparable with that after walking 3 min with elevated vacuum for cases 2 and 3 (Table 1, column 4). The differences were 0.0 percent for case 2 and 0.1 percent for case 3 . For case 4, limb fluid volume after $3 \mathrm{~min}$ of walking with elevated vacuum averaged 0.7 percent less than that after 3 min walking with suction. When vacuum was then reduced, fluid volume increased 0.5 percent.

For all three (cases 2, 3, and 4), peak-to-peak fluid volumes were less with elevated vacuum than with suction (Table 1, column 2). The differences between elevated vacuum and suction were 2.3 percent for case 2 $(4.3 \%-2.0 \%), 0.4$ percent for case $3(1.3 \%-0.9 \%)$, and 0.7 percent for case $4(3.6 \%-2.9 \%)$.

\section{Cases 5 and 6: Short-Term Users of Electronic Elevated Vacuum-Within-Session Comparison of Different Vacuum Pressures}

Cases 5 and 6 both used electronic vacuum systems for short-term intervals, approximately 4 wk. However, case 6 used the electronic vacuum unit regularly outside the lab, while case 5 used it intermittently. Both subjects had excessive redundant soft tissue in their residual limbs, particularly distally.

\section{Case 5}

This subject was a 25 yr old female. She had her amputation 3 yr prior as a result of traumatic injury and then had a surgical revision $2 \mathrm{yr}$ later to remove excessive redundant soft tissue. Her residual limb was $14 \mathrm{~cm}$ in length and was fleshy and bulbous with adherent tissue on the distal tibia. She had a history of inflamed fungus from gel liner use. She was $58 \mathrm{~kg}$ in mass, $160 \mathrm{~cm}$ in height, and a very active K-4 level ambulator, using her prosthesis for $16 \mathrm{~h} / \mathrm{d}$. She was a marathon racer and triathlete and underwent much residual limb volume reduction during long runs. Otherwise, her limb volume was stable. She regularly used a PTB endoskeletal prosthesis with a gel liner and lock-and-pin suspension and Renegade Ultralite Foot (Freedom Innovations). During high activity she used an Iceflex endurance sleeve (Össur) for auxiliary suspension. She was fitted with a new socket equipped with an electronic elevated vacuum system (e-Pulse, Otto Bock) and with a Seattle Foot by her regular prosthetist for this investigation but did not feel comfortable wearing it regularly because she was concerned about falling while at her waitressing job. Thus, she used the elevated vacuum system intermittently. She was tested with her regular prosthesis approximately $10 \mathrm{wk}$ prior to testing with the elevated vacuum system.

\section{Case 6}

This subject was a 34 yr old male and had his limb amputation as a result of traumatic injury 3 yr prior. His residual limb was $19 \mathrm{~cm}$ in length and was bulbous with redundant soft tissue and a prominent distal tibia. His residual limb had much hair and was very sensitive to pain. He was $102 \mathrm{~kg}$ in mass, $188 \mathrm{~cm}$ in height, and a K-3 level ambulator. He wore his prosthesis for approximately 15 h/d. He regularly used a PTB endoskeletal prosthesis with a gel liner and lock-and-pin suspension and a dynamic response foot (Freedom Innovations). During moderate or high physical activity, he added socks to accommodate residual limb volume reduction. He was tested first using his regular prosthesis. Then for this investigation, he was fitted by his regular prosthetist with a new socket equipped with an electronic elevated vacuum system (e-Pulse) with a Seattle LightFoot. He used that system exclusively for 3 wk before bioimpedance testing. 
SANDERS et al. Effects of elevated vacuum on residual limb fluid volume

Table 1.

Results from vacuum pressure changes made within test session. All data are expressed as percentage of initial fluid volume measured at outset of test session.

\begin{tabular}{|c|c|c|c|c|}
\hline $\begin{array}{c}\text { Subject and Vacuum } \\
\text { Condition }\end{array}$ & $\begin{array}{c}\text { Walking } \\
\text { Peak-to-Peak }\end{array}$ & $\begin{array}{c}\text { Volume During } \\
\text { Short-Term Walk }\end{array}$ & $\begin{array}{l}\text { Volume for Vacuum Setting } \\
\text { Change Low to High }\end{array}$ & $\begin{array}{l}\text { Volume for Vacuum Setting } \\
\text { Change High to Low }\end{array}$ \\
\hline \multicolumn{5}{|l|}{ Manual Vacuum } \\
\hline Case $2 \mathrm{~S}$ & 4.3 & -0.1 & 0.0 & NA \\
\hline Case $2 \mathrm{~V}$ & 2.0 & +1.2 & & \\
\hline Case $3 \mathrm{~S}$ & 1.3 & +0.3 & +0.1 & NA \\
\hline Case $3 \mathrm{~V}$ & 0.9 & +0.4 & & \\
\hline Case $4 \mathrm{~S}$ & 3.6 & -0.5 & -0.7 & +0.5 \\
\hline Case $4 \mathrm{~V}$ & 2.9 & -0.6 & & \\
\hline \multicolumn{5}{|l|}{ Electronic Vacuum } \\
\hline Case 5 1-setting EV & 3.2 & +0.2 & +0.6 & -0.2 \\
\hline Case 5 4-setting EV & 3.4 & +0.5 & & \\
\hline Case 6 1-setting EV & 1.8 & +0.6 & +1.9 & -1.0 \\
\hline Case 6 4-setting EV & 2.0 & +1.0 & & \\
\hline \multicolumn{5}{|c|}{$\begin{array}{l}\text { Blue = No to minimal difference with respect to its pair. } \\
\text { Green = Meaningful difference with respect to its pair, expected direction of change. }\end{array}$} \\
\hline \multirow{2}{*}{\multicolumn{5}{|c|}{$\begin{array}{l}\text { Black = No comparison was possible. } \\
\mathrm{EV}=\text { electronic vacumm } \mathrm{NA}=\text { not available because of in }\end{array}$}} \\
\hline & & & & \\
\hline
\end{tabular}

Protocol

After the electrodes were applied, the subject sat quietly for $2 \mathrm{~min}$. Each subject walked at a selected vacuum setting for $3 \mathrm{~min}$, first with the setting increased from one 3 min walk to the next. Four settings were possible on the electronic elevated vacuum unit, with " 4 " being the highest pressure (labeled as "60 $\mathrm{kPa}$ " by the manufacturer). The other settings were " 1 " (25 kPa), " 2 " (36 kPa), and "3” (48 kPa). We did not measure vacuum pressures during the studies; thus, the actual pressures achieved and their fluctuations during use were unknown. Pressure fluctuations during walking have been reported for other products [28]. The subject then sat quietly for $2 \mathrm{~min}$ and then walked again for $3 \mathrm{~min}$ at the same vacuum settings as before sitting. Then, the vacuum setting was decreased from one walk to the next. For case 5, two settings were used: the 1-setting and 4-setting (highest vacuum pressure). For case 6 , four settings were used: the 1-setting, 2-setting, 3-setting, and 4-setting. Case 5 preferred the 4setting, while case 6 preferred the 3-setting. Because the vacuum pump could be heard activating early on during each 3 min walk, we concluded that the elevated vacuum pressures during walks with the high settings were not maintained during subsequent walks at lower vacuum settings. In other words, the result that changes in limb fluid volume were small when vacuum pressure settings were reduced (Figure 4) was not a result of high vacuum pressure being maintained from the previous 3 min walk interval.
Results

When the vacuum pressure was increased from the 1setting to the 4-setting, peak-to-peak limb fluid volume changes did not change as much as they did for cases 2 , 3 , and 4 when the socket was changed from suction to manual elevated vacuum. Peak-to-peak differences for the 1-setting versus the 4 -setting averaged 0.2 percent for case $5(3.4 \%-3.2 \%)$ and 0.2 percent for case $6(2.0 \%-$ $1.8 \%)$.

Limb fluid volume increased more during the walks at the higher vacuum pressure setting than at the lower vacuum pressure setting. The difference in fluid volume increase during walks for the 4-setting compared with the 1 -setting was 0.3 percent for case $5(0.5 \%-0.2 \%)$ and 0.4 percent for case $6(1.0 \%-0.6 \%)$.

Limb fluid volume was greater at the end of walks at the 4-setting than at the 1-setting for both subjects (Table 1, column 4). The increase in fluid volume upon elevating the vacuum setting was greater than the decrease in fluid volume upon reducing the vacuum setting. For case 5, the fluid volume change from elevating the vacuum setting was 0.6 percent and the change for reducing the setting was -0.2 percent. For case 6 , the fluid volume change from elevating the vacuum setting (difference between 1-setting and 4-setting) was 1.9 percent and the change for reducing the vacuum setting was -1.0 percent. However, note that for case 6 , four vacuum settings were tested, unlike case 5 , for whom only two settings were tested. This difference in protocol meant that case 6 
walked longer using elevated vacuum, which might partially explain why case 6's fluid volume changes were higher than case 5's.

For case 6, increasing the vacuum setting from 1 to 2 to 3 to 4 showed a gradual increase in limb fluid volume, but decreasing the setting from 4 to 3 to 2 to 1 did not show a gradual fluid volume decrease (Figure 4, right panel). Instead, a sudden decrease only was seen for changing from the 2-setting to the 1-setting. No change in peak-to-peak fluid volume was observed for any pair of settings (walks at the same vacuum pressure setting) for either subject. From visual inspection of the fluid volume changes over time for both subjects (Figure 4), the limb fluid volume changes induced from changing the vacuum pressure appear to be superposed on a gradual limb fluid volume increase during the session.

\section{Cases 5, 6, and 7: Short-Term Users of Elevated Vacuum-Between-Session Comparison of Elevated Vacuum Versus Lock-And-Pin}

For cases 5 and 6 (described above), as well as case 7 (described below), we compared each subject's results using an elevated vacuum socket to results using a lockand-pin socket (different socket). The tests were conducted on different days. Data collection sessions for each subject were at approximately the same time of day for cases 5 and 6 but were at a different time of day for case 7 (Table 2, column 1). Vacuum settings on the units for cases 5 and 6 were the 3-setting and 4-setting, respectively.

\section{Case 7}

This subject was a 61 yr old male with type 2 diabetes. As a result of an unhealed neuropathic foot ulcer, he had a right transtibial amputation 8 yr prior. He was $163 \mathrm{~cm}$ tall, $99.5 \mathrm{~kg}$ in mass, and a K-2 level ambulator, using his prosthesis at least $12 \mathrm{~h} / \mathrm{d}$. He regularly used a PTB endoskeletal prosthesis with a gel liner and lockand-pin and a Fusion Foot (Ohio Willow Wood; Mt. Sterling, Ohio). His skin was thin and fragile with poor sensation, and in the past, he had had blisters and wounds on his residual limb. He did not typically add socks during the day to accommodate residual limb volume reduction, though the research practitioner considered this practice a reflection of his neuropathy. She believed that he did not sense his limb volume reduction and the need to add a sock. He underwent bioimpedance testing with his regular prosthesis first. He was then fitted with a new socket equipped with a P2 Harmony unit and Seattle Lightfoot.

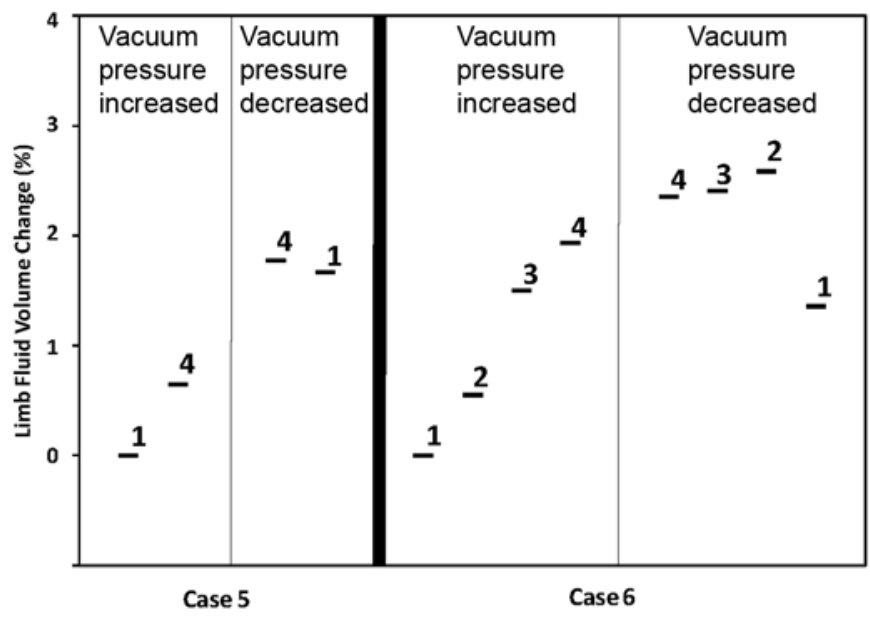

Figure 4.

Results for electronic elevated vacuum users: Cases 5 and 6. Limb fluid volumes at end of walks are shown. Numbers immediately above data lines are vacuum pressure settings. Limb fluid volume changes are relative to fluid volume after first walk at 1-setting.

He used this prosthesis exclusively for 3 wk before bioimpedance testing.

\section{Protocol}

The protocol for the session using the lock-and-pin suspension and the session using elevated vacuum was the same and was similar to that described for case 1 . After the electrodes were put on the residual limb and the prosthesis donned, the subject sat comfortably in a chair for 2 min. Then, the subject stood with equal weight bearing for $5 \mathrm{~min}$ and subsequently walked on the treadmill for 5 min. After sitting quietly for $2 \mathrm{~min}$, the subject again stood for $5 \mathrm{~min}$ with equal weight bearing and then walked on the treadmill for $5 \mathrm{~min}$. The subject then sat, doffed the prosthesis and liner, and sat quietly for $10 \mathrm{~min}$. The fluid volume change after doffing was calculated as the fluid volume after 10 min of sitting minus that measured immediately after the prosthesis and liner were removed. The fluid volume during standing right after the last walk minus the fluid volume at the outset of the first stand represented the session fluid volume change.

\section{Results}

The results showed subject-dependent trends. Peakto-peak residual limb fluid volume for elevated vacuum sockets compared with lock-and-pin sockets was higher for case 6 , lower for case 7 , and not different for case 5 
Table 2.

Results from pin and vacuum sockets. Test sessions were conducted on different days (month, day, and start time of test sessions are shown).

\begin{tabular}{|c|c|c|c|c|}
\hline $\begin{array}{c}\text { Subject, Date, Start Time, and } \\
\text { Type of Socket }\end{array}$ & Walking Peak-to-Peak & $\begin{array}{l}\text { Volume During } \\
\text { Short-Term Walk }\end{array}$ & Postdoff $10 \mathrm{~min}$ & Volume over Session \\
\hline Case 5, 1/25, 2:40 pm, PIN & 2.1 & +0.2 & +2.0 & -0.5 \\
\hline Case 5, 4/2, 3:15 pm, EV & 2.1 & +2.1 & +2.5 & +1.2 \\
\hline Case 6, 3/21, 2:20 pm, EV & 1.0 & +0.5 & +3.2 & +0.8 \\
\hline Case 7, 1/4, 10:00 am, PIN & 2.2 & -0.2 & +0.7 & -2.1 \\
\hline \multicolumn{5}{|c|}{$\begin{array}{l}\text { Blue = No to minimal difference with respect to its pair. } \\
\text { Green = Meaningful difference with respect to its pair, expected direction of change. } \\
\text { Red = Meaningful difference with respect to its pair, unexpected direction of change. } \\
\text { Black = No comparison was possible. }\end{array}$} \\
\hline
\end{tabular}

(Table 2, column 2). Fluid volume changes during 5 min walks were greater for elevated vacuum than for lockand-pin for case 5 and not different for cases 6 and 7 (Table 2, column 3). Fluid volume changes during 10 min after doffing for elevated vacuum were lower than for lock-and-pin for case 6 and higher for cases 5 and 7 (Table 2, column 4). For all three cases, the difference in fluid volume from the brief stand right after the last walk minus the fluid volume at the outset of the first stand was more positive for elevated vacuum than for lock-and-pin (Table 2, column 5). Thus, during the test session, subject limbs tended to increase more (or decrease less) for elevated vacuum compared with lock-and-pin.

\section{DISCUSSION}

Bioimpedance analysis provides insight into residual limb fluid volume changes while the prosthesis is worn. This capability allowed us to investigate whether for subjects using elevated vacuum, in-socket fluid volume change results were consistent with conclusions drawn from studies using predonning and postdoffing volume measurement differences [3-5].

Bioimpedance analysis has been validated against other techniques for fluid volume assessment. In whole body analysis, bromide dilution and bioimpedance extracellular fluid volume measurements were shown to be highly correlated $(r>0.9)$ [20]. Limb segment muscle volumes determined by magnetic resonance imaging were also shown to be highly correlated to bioimpedance measurements $(r>0.9)$ [24]. Our measurements of limb fluid volume change after doffing in the present study (median of 2.0\%) were less than those reported by Zachariah et al. using an optical scanning method on six subjects (median of 5.0\%) [29]. However, in Zachariah et al.'s study [29], measurements after doffing were taken during standing, which would be expected to increase volume changes compared with sitting, the postdoffing measurement position in the present investigation. Because bioimpedance measurements may be sensitive to movement of the voltage-sensing electrodes relative to each other, we took precautions here to avoid electrode movement detrimentally affecting interpretation of bioimpedance results. Differences in limb fluid volume were calculated only for like postures, e.g., during a walking interval, over a sitting interval (after doffing), from standing with equal weight bearing at one point in time to standing with equal weight bearing at another point in time. We assumed that like postures had similar electrode positions relative to each other.

\section{Did Limb Fluid Volume Maintain or Increase During Walking when Subjects Used Elevated Vacuum?}

Residual limb fluid volume increased during shortterm walks on all healthy subjects $(1,2,3,5,6)$ when elevated vacuum was used. However, limb fluid volume also typically increased during short-term walks when the vacuum was off or a lock-and-pin suspension was used instead. In other studies, we have similarly found that healthy subjects experienced limb fluid volume increases during short-term walks [27]. Our interpretation of these findings is that the increase in fluid volume during short-term walks is primarily a result of a rise in 
arterial blood pressure and arterial dilation, resulting in more blood flow in the residual limb and an increase in arterial to interstitial fluid transport. During short-term walks, these physiological changes may be more dominant than elevated vacuum toward increasing limb fluid volume. Limb fluid volume decreases during stands immediately before walks might have accentuated walking limb fluid volume increases in that they temporarily dehydrated the residual limb. An interesting and needed area of future investigation is bioimpedance monitoring of long-term walking to determine whether elevated vacuum has a more dominant role in that time frame.

The two subjects with compromised health, cases 4 and 7 , were the only subjects to demonstrate constant or decreasing residual limb volumes during walks, consistent with results from previous case studies [27]. Case 4 had peripheral arterial disease/peripheral vascular disease, and case 7 was diabetic with poor sensation in his residual limb. Koïtka et al. [30], McLellan et al. [31], and Fromy et al. [32] demonstrated that people with diabetes and sensory neuropathy tended to have a reduced capability for pressure-induced vasodilation compared with nondiseased subjects. Thus, for case 7 , a reduced arterial volume flow rate induced by a lack of pressure-injured vasodilation and, for case 4, reduced arterial flow resulting from arterial disease may explain why these subjects' limb fluid volume changes during walks were lower than those of healthy subjects.

Only one subject in the present study (case 1) was a regular long-term ( $>6 \mathrm{mo}$ ) user of elevated vacuum. His change in residual limb fluid volume over the session $(+2.1 \%)$ was much larger than that of other subjects. Further studies need to be conducted to determine whether this trend represents an adaptive response to elevated vacuum over long-term use.

The single subject who demonstrated a reduction in limb fluid volume during walks with both vacuum and suction sockets (case 4) was unusual in that his socket, designed to accommodate a neuroma at the anterior distal end of his residual limb, was wedge-shaped (conical) but with a localized relief distally. This socket design may have pushed the subject deeper into the socket when walking, and the wedging effect may have reduced limb fluid volume. This socket design is contrary to that suggested by companies marketing elevated vacuum products. Manufacturers recommend total contact with uniform pressure distally. A systematic study investigating the effects of socket shape on limb volume changes during elevated vacuum use is warranted and would aid understanding of how sensitive limb volume shifts are to socket shape.

For some, but not all, subjects, limb fluid volume increases during walks were greater with greater vacuum, consistent with expectation. The vacuum pulled soft tissues outward, helping to pull fluid into the interstitial space within the residual limb, which increased or maintained limb fluid volume. For no subject was limb fluid volume change during walks lower with greater vacuum. One possible reason why some of the subjects did not display limb fluid volume increases as large as others may have to do with the size difference between the socket and residual limb. If a socket were tight on the residual limb at the outset of testing, then the socket would restrict residual limb enlargement upon vacuum application. No or minimal limb volume increase would occur. A loose socket at the outset of the testing protocol, however, would allow the residual limb to enlarge when vacuum was applied. This expectation is consistent with Goswami et al.'s findings that limb volume increase was greater with elevated vacuum when an oversized socket was used compared with a normal or an undersized socket [4]. Thus, with a loose socket at the outset, a subject using elevated vacuum would be expected to experience limb fluid volume increase during short-term walks, but with a tight socket, no limb fluid volume increase would be expected.

\section{Did Limb Fluid Volume Increase when Vacuum was Turned from Off to On or Vacuum was Increased from Low to High Pressure Setting?}

Results depended on the type of elevated vacuum system used. For the two electronic elevated vacuum users (cases 5 and 6), limb fluid volume increased substantially after increasing vacuum pressure. The increases from the 1-setting to the 4-setting on the vacuum device were 0.6 percent for case 5 and 1.9 percent for case 6 . For the Harmony users, however, limb fluid volume increases when switching from suction to vacuum were low: 0.0, 0.1 , and -0.7 percent (cases 2,3 , and 4 , respectively).

While the heavy weight of the Harmony system compared with the electronic system probably contributed to these differences, we suspect that differences in residual limb soft tissue content between the two sets of subjects was also a dominant factor. The electronic elevated vacuum users (cases 5 and 6) had very fleshy limbs with much redundant soft tissue, unlike the Harmony users 
(cases 2, 3, and 4), who had bony residual limbs. More soft tissue may have enhanced the capability for fluid volume change.

The magnitudes of limb fluid volume change over the course of the session for manual elevated vacuum in the present study were much lower than limb volume changes reported by Board et al. [3]. Changes in the present investigation ranged from -1.6 to +1.2 percent, and in Board et al.'s study they ranged from -1.6 to +8.5 percent. Part of the reason for the inconsistency is that different measurements were taken. Limb fluid volume change was assessed in the present study, while Board et al. assessed total limb volume change. Further, we measured in-socket changes, while Board et al. measured outof-socket changes. Board et al. measured external limb shape before donning and after doffing by using a casting and water displacement method, and we used bioimpedance while the residual limb was within the socket. Further, the section of the limb we tested did not include the distal end, unlike Board et al. who included the entire residual limb. Our walking times totaled approximately 10 min over the 30 min test session, while Board et al.'s subjects walked continuously for $30 \mathrm{~min}$ and thus were more physically exerted. One of our subjects (case 7) had his amputation for dysvascular reasons, while all Board et al.'s subjects were traumatic injury or congenital amputees. Thus, numerous variables might have contributed to the substantial measurement differences between Board et al.'s and the present study.

In the present study, results from electronic elevated vacuum users showed that limb fluid volume increases from increasing vacuum pressure were of greater magnitude than limb fluid volume decreases from decreasing vacuum pressure (cases 5 and 6 ). This result suggests that resistance toward driving fluid out of the residual limb was greater than for bringing fluid into the limb. It is unclear whether this phenomenon was a result of using elevated vacuum, reflected the prior activity history, or was a physiological characteristic of these particular subjects. In terms of prescribing and adjusting vacuum pressure on individual users of elevated vacuum, these differences in fluid transport resistance are important to understand. Further investigation is needed to understand how elevated vacuum affects physiological fluid transport.

\section{Did Cyclic (Peak-to-Peak) Fluid Volumes During Walking Change when Vacuum was Activated?}

While the sampling rate of our system was lower than the walking rate of the subjects, we considered com- paring peak-to-peak fluid volumes for different test conditions acceptable because none of the subjects had a walking rate that was a multiple of the sampling rate $(1 \mathrm{~Hz})$. If the walking speed were a multiple of the instrument's sampling rate, then an aliasing problem with a consistent error in peak-to-peak limb fluid volume would occur, invalidating the peak-to-peak assessment.

The result in the present study that the peak-to-peak fluid volume decreased when subjects switched from suction to elevated vacuum (Harmony system) is consistent with expectation. Limb fluid volume changes within a step decreased with higher vacuum, presumably because there was less pistoning. Also possible is that the changes in peak-to-peak fluid volume reflect changes in muscle activation. Possibly subjects felt that the prosthetic socket was looser on the residual limb when elevated vacuum was off compared with on and, as a result, contracted their musculature more forcefully, inducing greater fluid volume change. Electromyography assessment would help evaluate this hypothesis.

Peak-to-peak fluid volumes did not decrease when the electronic elevated vacuum (e-Pulse system) was used and the vacuum was increased from the 1-setting to the 4-setting. This difference in result between the electronic and manual vacuum systems may have occurred because the change in vacuum pressure for the electronic system (1-setting to 4-setting) was likely less than the change in vacuum pressure for suction versus manual vacuum (Harmony system). It is also feasible that a relatively low threshold vacuum pressure was sufficient to substantially reduce pistoning in the electronic elevated vacuum sockets, and the 1-setting on the system (25 kPa according to manufacturer literature) was above this threshold. Note, however, that vacuum pressure was not measured in the present study; thus, actual pressures are unknown. Other possible explanations include that the manual system was much heavier than the electronic vacuum system, inducing greater pistoning and thus greater peak-to-peak limb fluid volume change when the vacuum pressure was increased; the electronic system applied continuous vacuum, unlike the manual system, where vacuum was applied intermittently (i.e., only during walking); and the electronic elevated vacuum users (cases 5 and 6) had much redundant soft tissue, unlike the Harmony users (cases 2, 3, and 4), who had relatively bony residual limbs. Too many variables exist to allow us to determine what caused differences in peak-to-peak walking fluid volume dependence on vacuum pressure for electronic versus manual systems. In the future, all 
the just-listed variables will need to be considered in studies designed to investigate peak-to-peak limb fluid volume changes during walking.

\section{Were Fluid Volume Changes Within Session Different Using Elevated Vacuum Sockets Versus Lock-and-Pin Sockets?}

All three subjects (cases 5, 6, 7) demonstrated more positive changes in limb fluid volume from the beginning to the end of the test session when using elevated vacuum compared with a lock-and-pin suspension socket. This result is consistent with Board et al. [3] and Gerschutz et al. [5]. Both researcher groups found greater volume increase from the beginning to the end of the session for elevated vacuum versus suction, though they measured out-of-socket volumes as opposed to in-socket volumes as assessed here. Unlike Gerschutz et al.'s results from a single subject [5], though, for two of the three subjects tested here during $10 \mathrm{~min}$ after doffing (cases 5 and 7), limb fluid volume increases were greater for elevated vacuum than for nonelevated vacuum. However, the present study was designed differently than Gerschutz et al.'s. In the present study, two different sockets were used, one with elevated vacuum and one with lock-andpin. The two sockets were not necessarily of the same volume. In Gerschutz et al.'s study, one socket, a consistent volume, was used at different vacuum settings (elevated vacuum, suction). These differences in study design might explain differences in the results. The small numbers of subjects further limits interpretation. The meaning of postdoffing limb volume change toward insocket volume change and subject well-being is a topic in need of further investigation.

The magnitude of limb fluid volume change over the session may depend on the health of the subject, similar to limb fluid volume changes during walking. In similar reports, we have noted that limb fluid volume changes over the session reflect subject health. In the present study, we expect that the reduced fluid volume change over the session for case 7 is almost certainly due to his poorer health status (diabetic, neuropathy) compared with cases 5 and 6 . However, it is noteworthy that this subject still demonstrated an effect of elevated vacuum. His fluid volume loss over the session using elevated vacuum was less than that using suction (Table 2, column 5), which is an encouraging result toward potential use of elevated vacuum on vascularly compromised subjects. However, much further testing is needed before clinical practice recommendations can be made.
Peak-to-peak fluid volume changes were not consistently larger when a lock-and-pin socket was used compared with elevated vacuum. However, for the subject showing trends opposite to those expected (case 7) (Table 2, column 2), larger peak-to-peak fluid volumes using vacuum than lock-and-pin, the two test sessions were conducted at different times of the day. For the lock-and-pin test day, data were collected in the morning, while for the elevated vacuum test day, data were collected in the afternoon. This was unlike cases 5 and 6, who had afternoon test days for both sessions. Experience testing other subjects has shown limb fluid volume changes over a session depend on the time of day of testing [33]. Morning session and afternoon session results were typically different. If, in the present study, case 7's residual limb was smaller in the afternoon and no accommodation was performed, then greater pistoning and thus greater peak-to-peak fluid volume changes would be expected. Also possible is that differences in results between subjects for elevated vacuum versus lock-andpin are a result of different socket designs. Further investigation is needed to distinguish the influence of elevated vacuum from the influence of time of day and of socketto-limb size on residual limb fluid volume change.

\section{Future Research}

The results from this series of case studies do not consistently demonstrate that elevated vacuum maintained or increased limb fluid volume nor do they consistently demonstrate that elevated vacuum had no effect. Instead, elevated vacuum maintained or increased limb fluid volume on six of the seven subjects and affected some measures of limb fluid volume change but not others.

Results from these cases suggest that in future research efforts evaluating elevated vacuum, researchers need to consider a number of study design variables that may influence limb volume change measurements. These variables need to be considered when test results between two different conditions (e.g., elevated vacuum vs suction) are compared. Variables include-

1. Time of day of test.

2. Size of residual limb relative to size of socket.

3. Use of elevated vacuum as the regular prosthesis (i.e., subject accommodation).

4. Subject health.

5. Time into session that measurements are made and ordering of interventions within a session.

6. Limb soft tissue mechanical consistency. 
7. Socket shape.

8. Weight differences between prostheses tested.

9. Time after doffing that measurements are taken (if outof-socket measurement technique used).

Research efforts directed toward identifying which individuals are good candidates for elevated vacuum and why will facilitate effective application of elevated vacuum technology to appropriate patients. Also helpful would be studies that facilitate the design of computer algorithms within elevated vacuum units to appropriately regulate the magnitude of vacuum pressure to maintain limb volume and good suspension without subjecting residual limb soft tissues to undue risk.

\section{CONCLUSIONS}

This series of case studies on seven subjects showed that some subjects demonstrated less decrease (or more increase) in limb fluid volume using sockets with elevated vacuum compared with suction sockets or lockand-pin suspension sockets, while others did not. Some measures of limb fluid volume changed consistently, while others did not. A number of variables may affect limb fluid volume change. When designing future research studies, investigators need to consider these variables in study design, particularly when comparing elevated vacuum to another socket design.

\section{ACKNOWLEDGMENTS}

\section{Author Contributions:}

Study concept and design: J. E. Sanders, K. J. Allyn.

Acquisition of data: D. S. Harrison, T. R. Myers, K. J. Allyn.

Analysis and interpretation of data: J. E. Sanders, D. S. Harrison,

T. R. Myers, K. J. Allyn.

Drafting of manuscript: J. E. Sanders.

Critical revision of manuscript for important intellectual content: K. J. Allyn.

Financial Disclosures: The authors have declared that no competing interests exist.

Funding/Support: This material was based on work supported by the National Institutes of Health (grant NIH R01HD060585).

Additional Contributions: Clinical assistance from Ryan Blanck, CPO, of the Northwest Prosthetic and Orthotic Clinic and the Seattle Department of Veterans Affairs prosthetics center is appreciated. Timothy R. Myers is now with Newberg Roadhouse LLC.

Institutional Review: Internal review board approval from the University of Washington Human Subjects Division was obtained before testing on any subjects, and informed consent was obtained before any study procedures were initiated.

Participant Follow-Up: The authors do not plan to inform participants of the publication of this study because contact information is unavailable.

\section{REFERENCES}

1. Caspers CA, inventor. Hypobarically-controlled artificial limb for amputees. United States patent US 5,549,709. 1996 Aug 27.

2. Street GM. Vacuum suspension and its effect on the limb. Orthop Tech. 2007;4:1-4.

3. Board WJ, Street GM, Caspers C. A comparison of transtibial amputee suction and vacuum socket conditions. Prosthet Orthot Int. 2001;25(3):202-9. [PMID: 11860094] http://dx.doi.org/10.1080/03093640108726603

4. Goswami J, Lynn R, Street G, Harlander M. Walking in a vacuum-assisted socket shifts the stump fluid balance. Prosthet Orthot Int. 2003;27(2):107-13. [PMID: 14571940$]$ http://dx.doi.org/10.1080/03093640308726666

5. Gerschutz MJ, Denune JA, Colvin JM, Schober G. Elevated vacuum suspension influence on lower limb amputee's residual limb volume at different vacuum pressure settings. J Prosthet Orthot. 2010;22(4):252-56.

http://dx.doi.org/10.1097/JPO.0b013e3181f903df

6. Van Loan MD, Withers P, Matthie J, Mayclin PL. Use of bioimpedance spectroscopy to determine extracellular fluid, intracellular fluid, total body water, and fat-free mass. Basic Life Sci. 1993;60:67-70. [PMID: 8110166]

7. Organ LW, Bradham GB, Gore DT, Lozier SL. Segmental bioelectrical impedance analysis: Theory and application of a new technique. J Appl Physiol. 1994;77(1):98-112. [PMID: 7961281]

8. Fuller NJ, Hardingham CR, Graves M, Screaton N, Dixon AK, Ward LC, Elia M. Predicting composition of leg sections with anthropometry and bioelectrical impedance analysis, using magnetic resonance imaging as reference. Clin Sci (Lond). 1999;96(6):647-57. [PMID: 10334971] http://dx.doi.org/10.1042/CS19980376

9. Salinari S, Bertuzzi A, Mingrone G, Capristo E, Scarfone A, Greco AV, Heymsfield SB. Bioimpedance analysis: A useful technique for assessing appendicular lean soft tissue mass and distribution. J Appl Physiol. 2003;94(4):1552-56. [PMID: 12626475]

10. Dittmar M. Reliability and variability of bioimpedance measures in normal adults: Effects of age, gender, and body mass. Am J Phys Anthropol. 2003;122(4):361-70.

[PMID: 14614757] http://dx.doi.org/10.1002/ajpa.10301 
11. Andreoli A, Melchiorri G, De Lorenzo A, Caruso I, Sinibaldi Salimei P, Guerrisi M. Bioelectrical impedance measures in different position and vs dual-energy X-ray absorptiometry (DXA). J Sports Med Phys Fitness. 2002; 42(2):186-89. [PMID: 12032414]

12. De Vries PM, Meijer JH, Vlaanderen K, Visser V, Oe PL, Donker AJ, Schneider H. Measurement of transcellular fluid shift during haemodialysis. Part 2. In vitro and clinical evaluation. Med Biol Eng Comput. 1989;27(2):152-58. [PMID: 2601434]

13. Zhu F, Schneditz D, Wang E, Martin K, Morris AT, Levin NW. Validation of changes in extracellular volume measured during hemodialysis using a segmental bioimpedance technique. ASAIO J. 1998;44(5):M541-45.

[PMID: 9804490]

http://dx.doi.org/10.1097/00002480-199809000-00045

14. Shulman T, Heidenheim AP, Kianfar C, Shulman SM, Lindsay RM. Preserving central blood volume: Changes in body fluid compartments during hemodialysis. ASAIO J. 2001;47(6):615-18. [PMID: 11730198] http://dx.doi.org/10.1097/00002480-200111000-00009

15. Donadio C, Consani C, Ardini M, Bernabini G, Caprio F, Grassi G, Lucchesi A, Nerucci B. Estimate of body water compartments and of body composition in maintenance hemodialysis patients: Comparison of single and multifrequency bioimpedance analysis. J Ren Nutr. 2005;15(3): 332-44. [PMID: 16007563] http://dx.doi.org/10.1016/j.jrn.2005.04.001

16. Cole KS, Li CL, Bak AF. Electrical analogues for tissues. Exp Neurol. 1969;24(3):459-73. [PMID: 5800962] http://dx.doi.org/10.1016/0014-4886(69)90149-6

17. De Lorenzo A, Andreoli A, Matthie J, Withers P. Predicting body cell mass with bioimpedance by using theoretical methods: A technological review. J Appl Physiol. 1997; 82(5):1542-58. [PMID: 9134904]

18. Grimnes S, Martinsen OG. Bioimpedance and bioelectricity basics. 2nd ed. Amsterdam (the Netherlands): Elsevier/ Academic; 2008.

19. Fenech M, Jaffrin MY. Extracellular and intracellular volume variations during postural change measured by segmental and wrist-ankle bioimpedance spectroscopy. IEEE Trans Biomed Eng. 2004;51(1):166-75. [PMID: 14723506] http://dx.doi.org/10.1109/TBME.2003.820338

20. Siconolfi SF, Gretebeck RJ, Wong WW, Pietrzyk RA. Suire SS. Assessing total body and extracellular water from bioelectrical response spectroscopy. J Appl Physiol. 1997; 82(2):704-10. [PMID: 9049756]

21. Segal KR, Burastero S, Chun A, Coronel P, Pierson RN Jr, Wang J. Estimation of extracellular and total body water by multiple-frequency bioelectrical-impedance measurement. Am J Clin Nutr. 1991;54(1):26-29. [PMID: 2058583]
22. Wotton MJ, Thomas BJ, Cornish BH, Ward LC. Comparison of whole body and segmental bioimpedance methodologies for estimating total body water. Ann N Y Acad Sci. 2000; 904:181-86. [PMID: 10865733] http://dx.doi.org/10.1111/j.1749-6632.2000.tb06444.x

23. Armstrong LE, Kenefick RW, Castellani JW, Riebe D, Kavouras SA, Kuznicki JT, Maresh CM. Bioimpedance spectroscopy technique: Intra-, extracellular, and total body water. Med Sci Sports Exerc. 1997;29(12):1657-63. [PMID: 9432101]

http://dx.doi.org/10.1097/00005768-199712000-00017 Erratum in: Med Sci Sports Exerc. 1998;30(7):1178.

24. Miyatani M, Kanehisa H, Masuo Y, Ito M, Fukunaga T. Validity of estimating limb muscle volume by bioelectrical impedance. J Appl Physiol. 2001;91(1):386-94. [PMID: 11408456]

25. Sanders JE, Rogers EL, Abrahamson DC. Assessment of residual-limb volume change using bioimpedance. J Rehabil Res Dev. 2007;44(4):525-36. [PMID: 18247249] http://dx.doi.org/10.1682/JRRD.2006.08.0096

26. Medicare region $C$ durable medical equipment prosthetics orthotic supplier (DMEPOS) manual. Columbia (SC): Palmetto GBA; 2005. p. 53.5-53.6.

27. Sanders JE, Harrison DS, Allyn KJ, Myers TR. Clinical utility of in-socket residual limb volume change measurement: Case study results. Prosthet Orthot Int. 2009;33(4): 378-90. [PMID: 19961297] http://dx.doi.org/10.3109/03093640903214067

28. Gerschutz MJ, Haynes ML, Colvin JM, Nixon D, Denune JA, Schober G. A vacuum suspension measurement tool for use in prosthetics research and clinical outcomes: Validation and analysis of vacuum pressure in a prosthetic socket. J Prosthet Orthot. 2010;22(3):172-76. http://dx.doi.org/10.1097/JPO.0b013e3181e8feaa

29. Zachariah SG, Saxena R, Fergason JR, Sanders JE. Shape and volume change in the transtibial residuum over the short term: Preliminary investigation of six subjects. J Rehabil Res Dev. 2004;41(5):683-94. [PMID: 15558398] http://dx.doi.org/10.1682/JRRD.2003.10.0153

30. Koïtka A, Abraham P, Bouhanick B, Sigaudo-Roussel D, Demiot C, Saumet JL. Impaired pressure-induced vasodilation at the foot in young adults with type 1 diabetes. Diabetes. 2004;53(3):721-25. [PMID: 14988257] http://dx.doi.org/10.2337/diabetes.53.3.721

31. McLellan K, Petrofsky JS, Zimmerman G, Lohman E, Prowse M, Schwab E, Lee S. The influence of environmental temperature on the response of the skin to local pressure: The impact of aging and diabetes. Diabetes Technol Ther. 2009;11(12):791-98. [PMID: 20001680] http://dx.doi.org/10.1089/dia.2009.0097

32. Fromy B, Sigaudo-Roussel D, Gaubert-Dahan ML, Rousseau P, Abraham P, Benzoni D, Berrut G, Saumet JL. 
Aging-associated sensory neuropathy alters pressureinduced vasodilation in humans. J Invest Dermatol. 2010; 130(3):849-55. [PMID: 19727122]

http://dx.doi.org/10.1038/jid.2009.279

33. Sanders JE, Allyn KJ, Harrison DS, Myers TR, Ciol MA. Are short-term limb fluid volume changes representative of diurnal changes? Proceedings of the American Academy of Orthotists and Prosthetists 37th Academy Annual Meeting and Scientific Symposium; 2011 Mar 16-19; Orlando, FL. p. F23

Submitted for publication November 17, 2010. Accepted in revised form April 27, 2011.
This article and any supplementary material should be cited as follows:

Sanders JE, Harrison DS, Myers TR, Allyn KJ. Effects of elevated vacuum on in-socket residual limb fluid volume: Case study results using bioimpedance analysis. J Rehabil Res Dev. 2011;48(10):1231-48.

DOI:10.1682/JRRD.2010.11.0219

ResearcherID: Joan Sanders, PhD: E-8204-2011 
